\title{
Together: Navy Reserve's Response to COVID-19 Pandemic
}

\author{
Eryn Hart Dutta, DO ${ }^{1} \quad$ Michael Barker, MD ${ }^{1} \quad$ Robert Gherman, MD ${ }^{1}$ \\ 1 US Navy Reserve, Bureau of Medicine and Surgery, Falls Church, \\ Virginia \\ Address for correspondence Robert Gherman, MD, 21636 \\ Ripplemead Drive, Laytonsville, MD 20882 \\ Am J Perinatol 2021;38:307-309. \\ (e-mail: ghermdoc@gmail.com).
}

\section{Abstract \\ Keywords \\ - COVID-19 \\ - pandemic \\ - ICU care \\ - critical care}

Under the direction of U.S. Northern Command for COVID-19 pandemic response efforts, approximately 500 Navy Reserve medical professionals were deployed to the New York City area from April to June 2020. Some of these providers were asked to serve in 11 overburdened local hospitals to augment clinic staffs that were exhausted from the battle against coronavirus. Two maternal/fetal medicine physicians were granted emergency clinical providers to assist in these efforts.

\section{Key Points}

- Maternal-fetal medicine physicians contributed significantly to the COVID-19 pandemic by managing ICU patients.

- Disparate, diverse medical professionals can pull together to form cohesive and functional teams.

- The Department of Defense can mobilize a large group of providers in a short amount of time.

Sitting down to eat dinner together, after completing our 12hour shift in an overflow COVID-19 intensive care unit (ICU) in New York City, we reflected on how we had arrived at this moment.

About 3 weeks ago, we had received a text at 1:13 Am: "You have Naval Reserve orders you need to complete. Travel tomorrow to Philadelphia airport. Buses from airport to Fort Dix." We packed a few bags worth of our basic military gear and were on the road in under 24 hours. Mixed, contradictory emotions enveloped us as we departed: excitement, disquietude sadness, and unease about our exact mission's intent. How would we be able to help and to what extent? "Why yes, I do critical care on pregnant patients, I am a maternal fetal medicine physician. I am here to help." We had previously deployed to combat zones, but somehow the imminent threat of personal danger was more tangible as we listed those dependents who would get our life insurance coverage.

"Good luck today. You are going to meet some truly exceptional people that need our help. Remember we are here to support what they need. Leave the egos at the door

received

August 1, 2020

accepted after revision

November 4, 2020

published online

December 10, 2020

and put on your humility cloak. They have been fighting every day since March 1st. We are like the cavalry, so let's help rescue them." We were under the initial impression that we would be working at the Javits Center, taking care of nonCOVID related medical conditions. Immediately after we arrived in New York City, we received our revised assignments; not only were we in the epicenter of COVID-19 infection, but we would be broken into small teams to run overflow ICUs in hard hit Brooklyn and Queens. The makeshift teams drew from specialties that included urology, bariatric, plastic, general and trauma surgery, internal medicine, and family practice. We were granted disaster privileges by the New York City Health + Hospitals System, which permitted us "to do everything possible within the scope of the license to save a patient's life or to save a patient from serious harm."

The day starts at 6:00 Am, boarding a van driven by a nurse or physician on their day off. It is about a 20 -minute ride. We are greeted daily by the hospital's administration thanking us for coming to work. We climb a generic aged stairway to the first of the personal protective equipment (PPE) rooms,

(c) 2020. Thieme. All rights reserved. Thieme Medical Publishers, Inc., 333 Seventh Avenue, 18th Floor, New York, NY 10001, USA
DOI https://doi.org/ 10.1055/s-0040-1721710. ISSN 0735-1631. 
placing ill-fitting scrubs on, in addition to the surgical cap, shoe covers, glasses, and face shields, depending on supplies. We then get report on how all the patients are doing. Who died on the last shift? No one? Fantastic. Who did and why? We don our PPE and begin rounds. What valiant attempts will be made today? The sad and frustrating part is that it does not seem to matter what ventilation adjustments or vasopressors are started or stopped at this stage of the disease. Do we start broad spectrum antibiotics again? Do we try another course of steroids? The overall theme is putting out fires and just keeping people alive until we cannot.

The first day on the unit was a success. One woman was extubated and a second gentleman self-extubated. We counted that as a win. They were shortly transferred. That same day a call came from a neighboring unit to assist a resident team as their patient was in bradycardia. Shortly after responding, they had a second patient that was experiencing a myocardial infarction and going into renal failure. Our daily routine involves managing heavy pulmonary secretions, more renal failure, electrolyte imbalances, hemorrhage, and thrombosis. Is it time to prone the patient yet?

We also watched patients die. Alone. Ravaged. This is very difficult as the face shield becomes streaked with beads of sweat after performing chest compressions in full PPE. The patient was a 54-year-old male, with comorbidities of hypertension, obesity, and diabetes mellitus, who had just been recently intubated. Regretfully, this had not been the first death we had experienced that night; despite the best efforts of our team of experienced physicians, the COVID virus had already emerged victorious twice before. Once a patient is discharged or dies, we get another transfer from the floor almost immediately. The next admission was a 45-year-old female, with poorly controlled type II diabetes mellitus and morbid obesity (easy day, as this is the bread and butter maternal-fetal medicine patient). She came in intubated as she had failed bilevel positive airway pressure. The radial arterial line and a femoral vein triple lumen catheter were placed. She had received steroids and her blood sugars were in the 400s. She received hydroxychloroquine and azithromycin, convalescent plasma, and was even enrolled in the remdesivir trial. She then developed a pseudomonas pneumonia. Vasopressors were stopped and started.

Another patient had been in the unit for several days but then rapidly declined. She had had numerous cardiac assaults in the few days leading up to her demise. Ultimately, she became oliguric. Her extremities became cold. The resident contacted the family and talked to them about her changed condition over the phone. They ultimately elected comfort care. We stopped the norepinephrine, she died 30 minutes later. That day her husband would be buried, the same disease killed them both. We helped the nurse prep her body for the morgue. We gently placed her in the body bag. She was taken to the morgue and a new patient was transferred within a few hours of her bed being empty.

A male patient presented from the floor. He was doing reasonably well on the ward until he went into the bathroom and desaturated and essentially went into respiratory arrest. He was intubated and sent to us. Upon arrival, he had mottled lower extremities. The only other time I had seen this was in the recently deceased. This man was alive. He had absent pedal and popliteal pulses. He was diaphoretic, soaking the entire bed easily 6 inches on each side of his body, and the sweat pooling on his neck. His facial hair was overgrown. As I looked at him, I thought of those I loved around his same age, I quickly looked away and focused on how we could help. The resident attempted to place a triple lumen catheter in the femoral vein. When she placed the line the blood that flowed out was mahogany, upon touching the drape a clot formed. This was far more impressive than what I see in pregnancy (clot formation in less than 2 seconds). This man's D-dimer was over $50,000 \mathrm{ng} / \mathrm{mL}$. He died a few hours later.

A recent patient death involved calling a patient's family over Facetime to prep them for their loved one's impending death. I had predicted an hour and he was gone 40 minutes later. I called the family back, where only one spoke English and he had to convey this message to his loved ones. As maternal/fetal medicine physicians, we relate bad news multiple times each day. We do not, however, give it over the phone where a family member is unable to say goodbye and hold their loved one's hand or convey serious mortality risk over an electronic device. At the conclusion of the brief discussion, the family member thanked us, humbling.

Reflecting on the 2-month experience, we realized that as maternal-fetal medicine physicians we contributed significantly to this pandemic. After crash refresher "on the job" training in COVID-19 critical care, learning about ventilator settings, sedation infusions, and vasopressors, it was amazing how disparate, diverse medical professionals pulled together to form cohesive, and functional teams. We have willingly sacrificed time, talents, and health for fellow human beings. Before coming to New York City, our biggest concerns centered around our kids being out of school and missed outings. However, seeing firsthand what our fellow medical providers had to deal with daily puts many of our shallow concerns in a bigger perspective.

When reflecting on lessons for the next pandemic, strengths include this was the first time that the Navy mobilized nearly 200 practitioners and nurses within 48 hours. It has shown that the department of defense can move a large group of providers in a short amount of time. This will hopefully shorten the time that a response can be mobilized in the setting of another pandemic. Instant teams developed across all specialties. The state of New York did an amazing job getting the providers and nurses credentialed and ready to practice. Emergency privileges were initiated in many instances in 24 to 48 hours. In addition, multiple physician specialties were utilized to perform in a setting outside their routine jobs. One could ask was it a wise decision to send providers and nurses into situations where they are not the most skilled for the job. In an ideal world, this would not occur. However, in the early days of COVID, none of us were experienced. We were more than capable and qualified to serve in the ICU setting, as we have had critical 
care experience. There were not enough critical care physicians to manage the number of patients. In addition, the medical recommendations seemed to be constantly changing daily. Maybe because this was due to the emerging nature of COVID-19, but it would have been helpful to have had a clear set of guidelines under which to operate (current best practices). Some hospitals we served in, had these, others did not.

But COVID-19 does not follow the rules and we do not know how to truly treat these sick patients. This disease is brutal-cytokine storms, cerebellar infarcts, huge femoral thromboses, renal failure, strokes, and mesenteric ischemia ravage those who might survive the initial respiratory insult. We have limited evidence that a treatment may work, but then it does not. We try something else. A few patients do get better, but most of those in our overflow ICUs are simply in line to die. We walk around adjusting vents, pressors, and fluids. You know why? Because doctors need to fix things. We cannot just sit and wait. We prepare for the next family that we will have to give a life altering phone call that will forever be changed by an enemy we are presently unable to conquer.

Note

The views expressed in this article are those of the author (s) and do not necessarily reflect the official policy or position of the Department of the Navy, Department of Defense, or the United States Government. Eryn H. Dutta, DO, Michael Barker, MD, and Robert Gherman, MD are military service members.

This work was prepared as part of their official duties. Title 17, USC, §105 provides that "Copyright protection under this title is not available for any work of the U.S. government." Title 17, USC, §101 defines a U.S. government work as a work prepared by a military service member or employee of the U.S. government as part of that person's official duties.

Conflict of Interest

None declared. 